\title{
Water shortage risk assessment using spatiotemporal flow simulation
}

\author{
Hsin-I Hsieh ${ }^{1}$, Ming-Daw Su' ${ }^{1}$ Yii-Chen Wu ${ }^{1}$ and Ke-Sheng Cheng ${ }^{1,2^{*}}$
}

\begin{abstract}
Paddy irrigation practices in Taiwan utilize complicated water conveyance networks which draw streamflows from different tributaries. Characterizing and simulating streamflow series is thus an essential task for irrigation risk assessment and planning mitigation measures. It generally involves modeling the temporal variation and spatial correlation of streamflow data at different sites. Like many other environmental variables, streamflows are asymmetric and non-Gaussian. Such properties exacerbate the difficulties in spatiotemporal modeling of streamflow data. A stochastic spatiotemporal simulation approach capable of generating non-Gaussian ten-day period streamflow data series at different sites is presented in this paper. Historical flow data from different flow stations in southern Taiwan were used to exemplify the application of the proposed model. Simulated realizations of the spatiotemporal anisotropic multivariate Pearson type III distribution were validated by comparing parameters and spatiotemporal correlation characteristics of the simulated data and the observed streamflow data. Risks of irrigation water shortage were estimated and the effect of mitigation measures was assessed using the simulated data.
\end{abstract}

Keywords: Spatiotemporal simulation, Risk assessment, Irrigation, Semi-variogram

\section{Background}

Rice is the most important staple food in Taiwan and many other countries in East and Southeast Asia. A significant proportion of the available water is utilized for paddy irrigation. In Taiwan, several irrigation associations are responsible for the management and operation of paddy irrigation. Generally, there are two crops of paddy rice in one year. The summer crop begins in late July and is harvested in late November, whereas the winter crop begins in mid-February and is harvested in late June. While typhoons can draw rainfalls for irrigation of the summer crop, the early stage of the winter crop falls in the dry season (from November to April of the next year) and is often faced with irrigation water shortage. The situation can get even worse when prolonged droughts occur. For example, in 2004 a severe drought which prolonged from January to June led to a fallow decision for most of the Jianan Irrigation District (JID) in southern Taiwan. Decision on whether mitigation

\footnotetext{
*Correspondence: rslab@ntu.edu.tw

${ }^{1}$ Department of Bioenvironmental Systems Engineering, National Taiwan

University, Taipei, Taiwan

Full list of author information is available at the end of the article
}

practices should be taken or what measures need to be implemented must be made in the very early stage of a severe drought or even prior to paddy transplanting. In coping with droughts, different practices have been adopted by irrigation associations including irrigation with reduced rate, scheduling rotational irrigation, and leaving paddy field fallow. However, due to the random nature of rainfall occurrences and their amounts, which leads to the spatiotemporal variability of stream flows, success of such decisions cannot be guaranteed and government agencies are often criticized for failing to take timely and appropriate mitigation practices or, sometimes, for making false-alarm decisions. In particular, the same mitigation practice may be effective in one year, yet may fail in another year due to different meteorological and hydrological conditions.

There exist a few time series models for flow simulations and water resources management (Fernandez and Salas 1990; Machiwal and Jha 2006). Most of these models or simulations were applied to cases which did not involve multi-site correlation and/or spatiotemporal correlation. For example, Fernandez and Salas (1990) developed a gamma time series simulation model and 
applied the proposed model to annual streamflow series of several rivers. To the best our knowledge, no models which take into account both the spatial and temporal variabilities of streamflows are available for planning drought mitigation measures in Taiwan. Thus, there is an urgent need for developing models or tools that can be used for probabilistically assessing and comparing the effectiveness of different potential mitigation measures in advance.

Water management for paddy irrigation usually involves utilization of water available from different sources including river flow at different locations and water storage in ponds and reservoirs. Within an irrigation district, available irrigation water of different tributaries can also be mutually conveyed to cope with severe droughts. Channels, ponds, reservoirs, and other flow intake and diversion structures together form a complex irrigation network. Success of irrigation management during droughts requires characterizing the spatiotemporal variation of streamflows at different stations and tools which can be used for assessing the effects of mitigation measures. Thus, the objectives of this study are twofold: (1) to develop a stochastic model which is capable of characterizing the spatiotemporal variability of multi-site streamflows within an irrigation district and (2) to demonstrate that a probabilistic risk assessment of potential mitigation practices can be conducted using the spatiotemporal streamflow simulation model.

This paper is organized as follows. The Background section provides a general description of the study area, the problems and difficulties in drought mitigation decision making, and objectives of this study. The Methods section gives a detailed account of the theories, including standardization of the streamflows, modeling standardized streamflows by an $L$-moments-based goodness-of-fit test, characterizing the spatiotemporal variation of the standardized streamflows by an anisotropic semi-variogram modeling, and sequential non-Gaussian random field simulation. The Results and Discussion section gives a rigorous assessment of the results of spatiotemporal streamflow simulations and also provides an exemplar application of irrigation water shortage risk assessment. The Conclusions section draws concluding remarks.

\section{Methods}

The Jianan Irrigation District which encompasses an area of $758 \mathrm{~km}^{2}$ in southern Taiwan (see Fig. 1) was selected as the study area. The Jianan Irrigation Association manages a complex irrigation network, which comprises reservoirs, irrigation canals, channels, ditches, flow diversion works, check dams, intake structures, etc., to regulate the amounts of irrigation water to be conveyed to individual paddy plots throughout the crop growth period. In
Taiwan, irrigation scheduling and the practices of irrigation water management are operated on a nominal tenday period (TDP) basis (Cheng et al. 2000a, b). Decisions on the amounts of water to be supplied to individual paddy plots are evaluated at the end of every TDP. Also, reservoir water managements are based on TDP-based rule curves. Each month is divided into three TDPs, with a total of 36 TDPs yearly. Twenty-six years (1975-2000) of TDP flow data available at 12 flow stations were collected. The required volumes of irrigation water are withdrawn from rivers and reservoirs through intake structures. River flow stations upstream of major water intake structures are listed in Table 1. TDP streamflows exhibit significant seasonal variation with low-flow season (from November to April of the next year) accounts for only approximately $13 \%$ of the annual total flow volume (Hsieh et al. 2014). For the sake of convenience, TDP streamflows will be referred to as streamflows or flows hereinafter.

\section{Characterizing spatiotemporal characteristics of streamflows}

In addition to the seasonal variation, streamflows at different locations also exhibit significant spatial correlation, even though most flow stations belong to different rivers or tributaries. Spatial correlation of streamflows arose from the fact that the amounts of rainfalls falling within the tributaries of individual flow stations are correlated. Therefore, a complete characterization of streamflows at different locations must take into account marginal distributions of streamflows at individual stations and their temporal and spatial correlations.

In addition to the seasonal variation, streamflows are higher at stations with larger drainage areas (see Fig. 2). In order to remove such seasonal and geophysical effects, observed flows at individual sites were standardized with respect to site-specific long-term averages and standard deviations, i.e.,

$$
X_{s}(i, j, k)=\frac{X(i, j, k)-m(i, j)}{s(i, j)}
$$

where $X(i, j, k)$ and $X_{s}(i, j, k)$ represent the observed and standardized flows of the $j$-th TDP period $(j=1,2, \ldots$, $36)$ in the $k$-th year at the $i$-th flow station, and $m(i, j)$ and $s(i, j)$ are the long-term average and standard deviation of the $j$-th TDP period flows at the $i$-th flow station, respectively. Thus, standardized flows at different sites and of different TDP periods are considered as random variables with zero expectation and unit standard deviation. Standardization of streamflows removes the heterogeneity of the expected value and variance of streamflows in the spatial (different sites) and temporal (different TDPs) domains. 


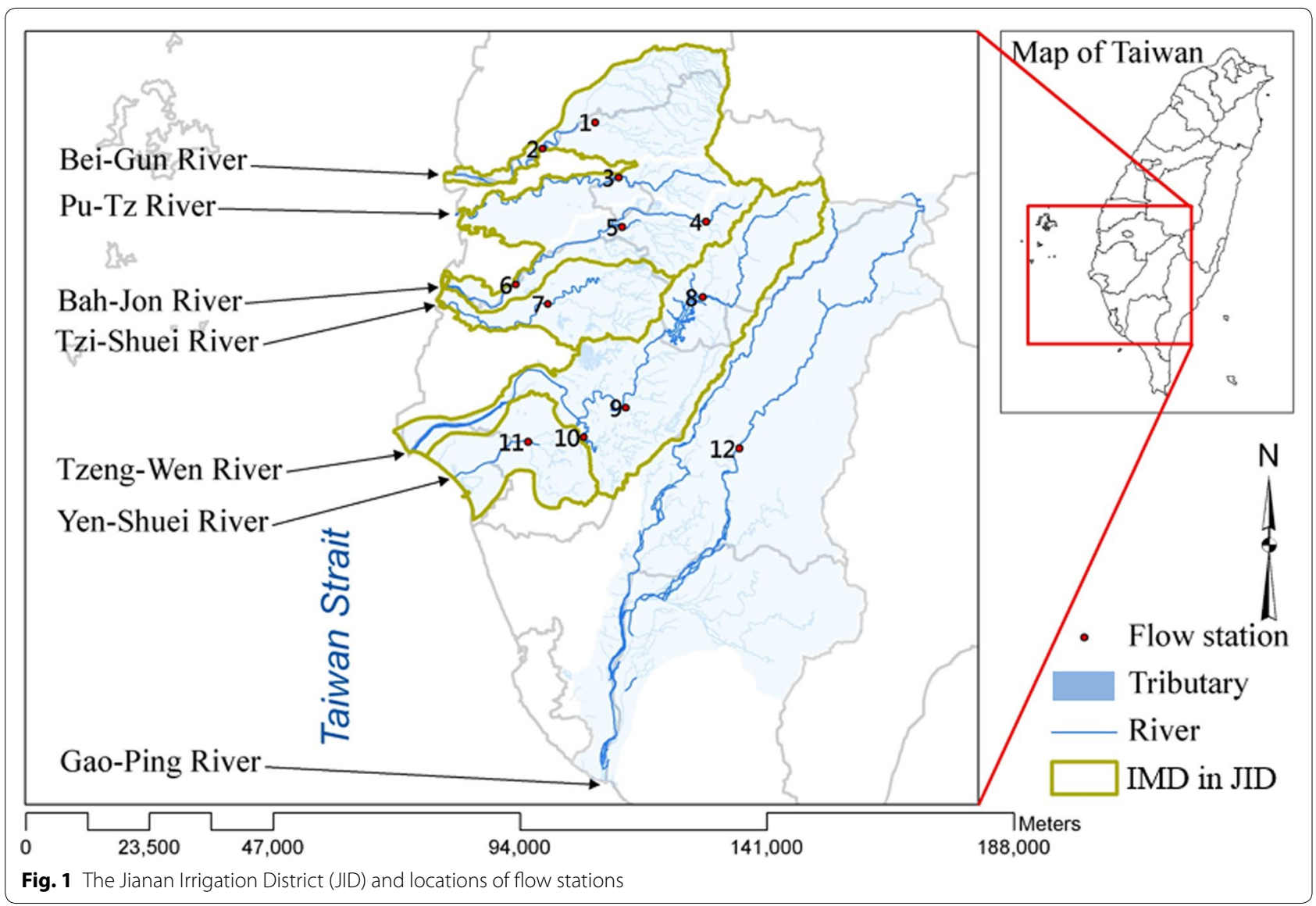

Table 1 River flow stations and their properties

\begin{tabular}{|c|c|c|c|c|}
\hline Station ID & River & $\begin{array}{l}\text { Irrigation Management } \\
\text { District (IMD) }\end{array}$ & $\begin{array}{l}\text { Drainage } \\
\text { area }\left(\mathrm{km}^{2}\right)\end{array}$ & $\begin{array}{l}\text { Long-term average } \\
\text { annual flow }\left(10^{6} \mathrm{~m}^{3}\right)\end{array}$ \\
\hline $1^{\mathrm{a}}$ & Bei-Gun & IMD-1 & 129.38 & 136.32 \\
\hline 2 & Bei-Gun & IMD-1 & 597.46 & 771.05 \\
\hline $3^{a}$ & $\mathrm{Pu}-\mathrm{Tz}$ & IMD-1 & 149.68 & 246.92 \\
\hline $4^{a}$ & Bah-Jon & IMD-1 & 83.15 & 181.93 \\
\hline $5^{b}$ & Bah-Jon & IMD-1 & 101.09 & 185.16 \\
\hline 6 & Bah-Jon & IMD-1 & 441.02 & 654.61 \\
\hline $7^{a}$ & Tzi-Shuei & IMD-2 & 226.66 & 300.01 \\
\hline $8^{a, b}$ & Tzeng-Wen & IMD-3 & 481.00 & 1070.19 \\
\hline $9^{a}$ & Tzeng-Wen & IMD-3 & 160.53 & 315.66 \\
\hline $10^{\mathrm{a}}$ & Tzeng-Wen & IMD-3 & 121.31 & 184.36 \\
\hline $11^{\mathrm{a}}$ & Yen-Shuei & IMD-4 & 146.46 & 176.00 \\
\hline 12 & Gao-Ping & & 812.03 & 1990.93 \\
\hline
\end{tabular}

a Upstream of water intake structures

b Reservoir inflow station

$L$-moments-based goodness-of-fit (GOF) tests were conducted for selection of appropriate distributions for standardized flows of individual TDPs at different stations. The $L$-moments-based GOF tests were based on the $95 \%$ acceptance regions of the sample linear moment ratios defined as follows. 


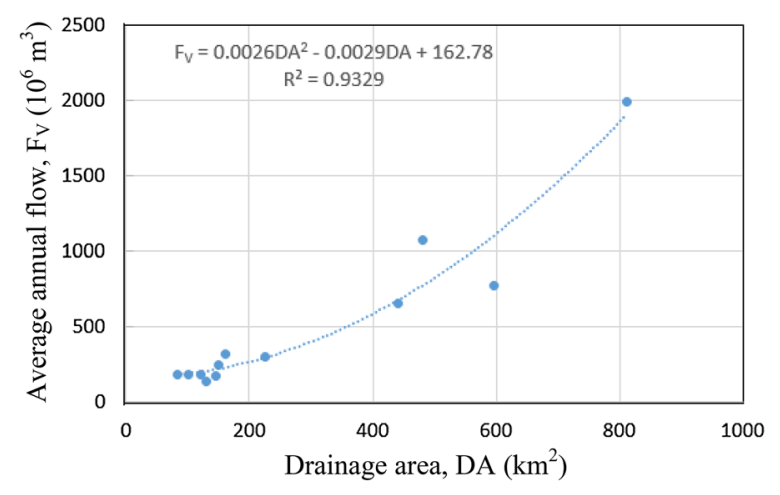

Fig. 2 Empirical relationship between the average annual flows $\left(F_{V}\right)$ and drainage areas (DA) of the 12 flow stations used in this study

For a random variable $X$ with quantile function $x(u)$, the $L$-moments can be expressed in terms of the probability weighted moments defined by Greenwood et al. (1979), and the first four $L$-moments are given by (Hosking and Wallis 1997)

$$
\begin{aligned}
& \lambda_{1}=\beta_{0} \\
& \lambda_{2}=2 \beta_{1}-\beta_{0} \\
& \lambda_{3}=6 \beta_{2}-6 \beta_{1}+\beta_{0} \\
& \lambda_{4}=20 \beta_{3}-30 \beta_{2}+12 \beta_{1}-\beta_{0},
\end{aligned}
$$

where $\beta_{r}, r=0,1,2, \cdots$ are probability weighted moments defined by

$$
\beta_{r}=\int_{0}^{1} x(u) u^{r} d u
$$

In terms of linear combinations of order statistics, the $L$-moments can also be expressed by

$$
\begin{aligned}
& \lambda_{1}=E\left(X_{1: 1}\right) \\
& \lambda_{2}=\frac{1}{2} E\left(X_{2: 2}-X_{1: 2}\right) \\
& \lambda_{3}=\frac{1}{3} E\left(X_{3: 3}-2 X_{2: 3}+X_{1: 3}\right) \\
& \lambda_{4}=\frac{1}{4} E\left(X_{4: 4}-3 X_{3: 4}+3 X_{2: 4}-X_{1: 4}\right) \\
& \tau_{r}=\lambda_{r} / \lambda_{2}(r=3,4)
\end{aligned}
$$

Given a random sample $\left\{x_{1}, x_{2}, \cdots, x_{n}\right\}$, an unbiased estimator of $\beta_{r}$ is given by

$$
b_{r}=\frac{1}{n} \sum_{j=r+1}^{n} \frac{(j-1)(j-2) \cdots(j-r)}{(n-1)(n-2) \cdots(n-r)} x_{j: n}
$$

where $x_{j: n}$ is the $j$-th order statistic from a random sample of size $n$. The sample $L$-moments $\left(\ell_{r}\right)$ and sample $L$-moment ratios $\left(t_{r}\right)$ can then be calculated by

$$
\begin{aligned}
& \ell_{1}=b_{0} \\
& \ell_{2}=2 b_{1}-b_{0} \\
& \ell_{3}=6 b_{2}-6 b_{1}+b_{0} \\
& \ell_{4}=20 b_{3}-30 b_{2}+12 b_{1}-b_{0} \\
& t_{r}=\ell_{r} / \ell_{2}(r=3,4)
\end{aligned}
$$

In the above equation, $t_{3}$ and $t_{4}$ are known as the sample $L$-skewness and $L$-kurtosis, respectively. The samplesize-dependent $95 \%$ acceptance regions of the sample linear moment ratios $\left(t_{3}, t_{4}\right)$ for different probability distributions including the Gaussian, Gumbel, and Pearson type III (PT3) distributions have been established (Liou et al. 2008; Wu et al. 2012). The null hypothesis that $\left\{x_{1}, x_{2}, \cdots, x_{n}\right\}$ originated from the specified distribution (for example, the Pearson type III) is rejected if and only if $\left(t_{3}, t_{4}\right)$ falls outside of their acceptance region of the specified distribution.

For each flow station, sample linear moment ratios $\left(t_{3}\right.$, $t_{4}$ ) of the standardized flows of individual TDPs were calculated. Using Station 3 and Station 8 as examples (see Fig. 3), for most TDPs, $\left(t_{3}, t_{4}\right)$ of the standardized flows fall within the $95 \%$ acceptance region (with respect to sample size $n=26$ ) of $L$-moment-ratio diagram (LMRD) of the Pearson type III distribution. It is apparent that the Gumbel and Gaussian distributions are not good choices for standardized flows. A few TDP-specific standardized flows which do not fall within the Pearson type III acceptance region are associated with very high (exceeding 3.0) coefficients of skewness. Through stochastic simulation, Wu et al. (2012) demonstrated that random samples from Pearson type III distributions with high coefficients of skewness tend to fall above the $95 \%$ acceptance regions in the $L$-moment-ratio diagram. Hence, in this study the standardized flows at different sites are modeled by a common Pearson type III distribution having a marginal density with zero expectation and unit variance.

The Pearson type III distribution has the following probability density:

$f_{X}(x ; \alpha, \beta, \varepsilon)=\frac{1}{\alpha \Gamma(\beta)}\left(\frac{x-\varepsilon}{\alpha}\right)^{\beta-1} e^{-(x-\varepsilon) / \alpha}, \quad \varepsilon \leq x<+\infty$

where $\alpha, \beta$, and $\varepsilon$ are, respectively, the scale, shape, and location parameters. Parameters of the Pearson type III distribution of the standardized flows were estimated by the method of $L$-moments. 


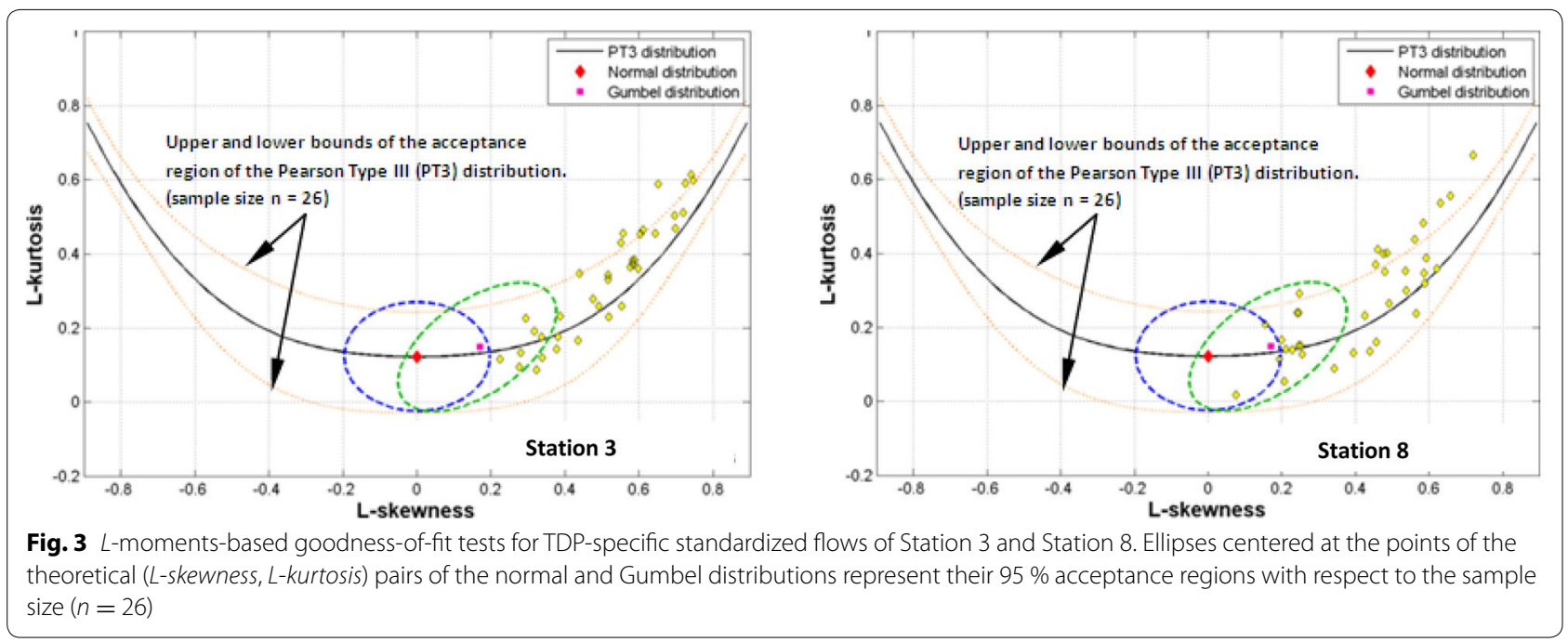

Hosking and Wallis $(1995,1997)$ showed that, for the Pearson type III distribution, the $L$-moments and distribution parameters $(\alpha, \beta$, and $\varepsilon)$ are related by the following equations:

$$
\begin{aligned}
& \lambda_{1}=\varepsilon+\alpha \beta \\
& \lambda_{2}=\frac{\pi^{-1 / 2} \alpha \cdot \Gamma\left(\beta+\frac{1}{2}\right)}{\Gamma(\beta)} \\
& \tau_{3}=6 I_{1 / 3}(\beta, 2 \beta)-3
\end{aligned}
$$

where $I_{x}(p, q)$ denotes the incomplete beta function ratio. By substituting the sample $L$-moments $\left(\ell_{1}, \ell_{2}, t_{3}\right)$ for $\left(\lambda_{1}\right.$, $\left.\lambda_{2}, \tau_{3}\right)$ in the above equations, the methods of $L$-moments estimates of the three parameters were calculated as $\hat{\alpha}=1.11, \hat{\beta}=0.745$ and $\hat{\varepsilon}=-0.826$. The spatial and temporal variations of the standardized flows were then investigated through the semi-variogram analysis.

A semi-variogram is a function that describes the spatial correlation structure of a random field. Let $Z(x)$ be a random variable at location $x$ and $\{Z(x), x \in \Omega\}$ be a homogeneous random field over a spatial domain $\Omega$. In geostatistics, the spatial variation of a random field is often expressed in terms of the semi-variogram $\gamma\left(x, x^{\prime}\right)$ defined as

$$
\gamma\left(x, x^{\prime}\right)=\frac{1}{2} E\left[\left(Z(x)-Z\left(x^{\prime}\right)\right)^{2}\right], x, x^{\prime} \in \Omega
$$

where $E(Z(x))$ represents the expected value of the random variable $Z(x)$. For a homogeneous random field, the semi-variogram is independent of the locations of $x$ and $x^{\prime}$ and can be expressed by $\gamma(h)$ with $h$ being the distance between $x$ and $x^{\prime}$.
A semi-variogram is a monotonic increasing function of the distance between $x$ and $x^{\prime}$, and can be characterized by its sill and range. The sill represents the asymptotic value of the semi-variogram and is numerically equal to $\sigma_{Z}^{2}$ the variance of $Z(x)$. The range is the minimum distance of $h$ beyond which two random variables $Z(x)$ and $Z\left(x^{\prime}\right)$ become independent. A commonly used exponential semi-variogram model has the following form (Journel and Huijbregts 1978; Cheng et al. 2003):

$$
\gamma(h)=\omega\left[1-e^{-(h / a)}\right]
$$

where $\omega$ represents the sill and $3 a$ is the practical range. For a homogeneous random field, the covariance function and semi-variogram are related by the following equation:

$$
\gamma(h)=C(0)-C(h),
$$

where $C\left(h=\left|x-x^{\prime}\right|\right)=\operatorname{Cov}\left(Z(x), Z\left(x^{\prime}\right)\right)$ is the covariance function. In our study, the spatiotemporal random field $\{Z(x), x \in \Omega\}$ represents the standardized flows at different locations or TDPs. Readers are referred to Journel and Huijbregts (1978) and Cheng et al. (2003) for details of the properties and modeling of the semi-variogram.

The spatiotemporal data structure of the standardized flow data, which are available at twelve stations for a period of 26 years (1975-2000), is demonstrated in Fig. 4. In our study, the spatiotemporal random field of the standardized flows is defined at 12 flow stations in the spatial domain and 36 TDPs in the temporal domain. Thus, a set of TDP-specific standardized flows (from the first TDP to the 36th TDP) observed at 12 flow stations represent a single realization of the spatiotemporal random field. Our stochastic spatiotemporal simulation of 


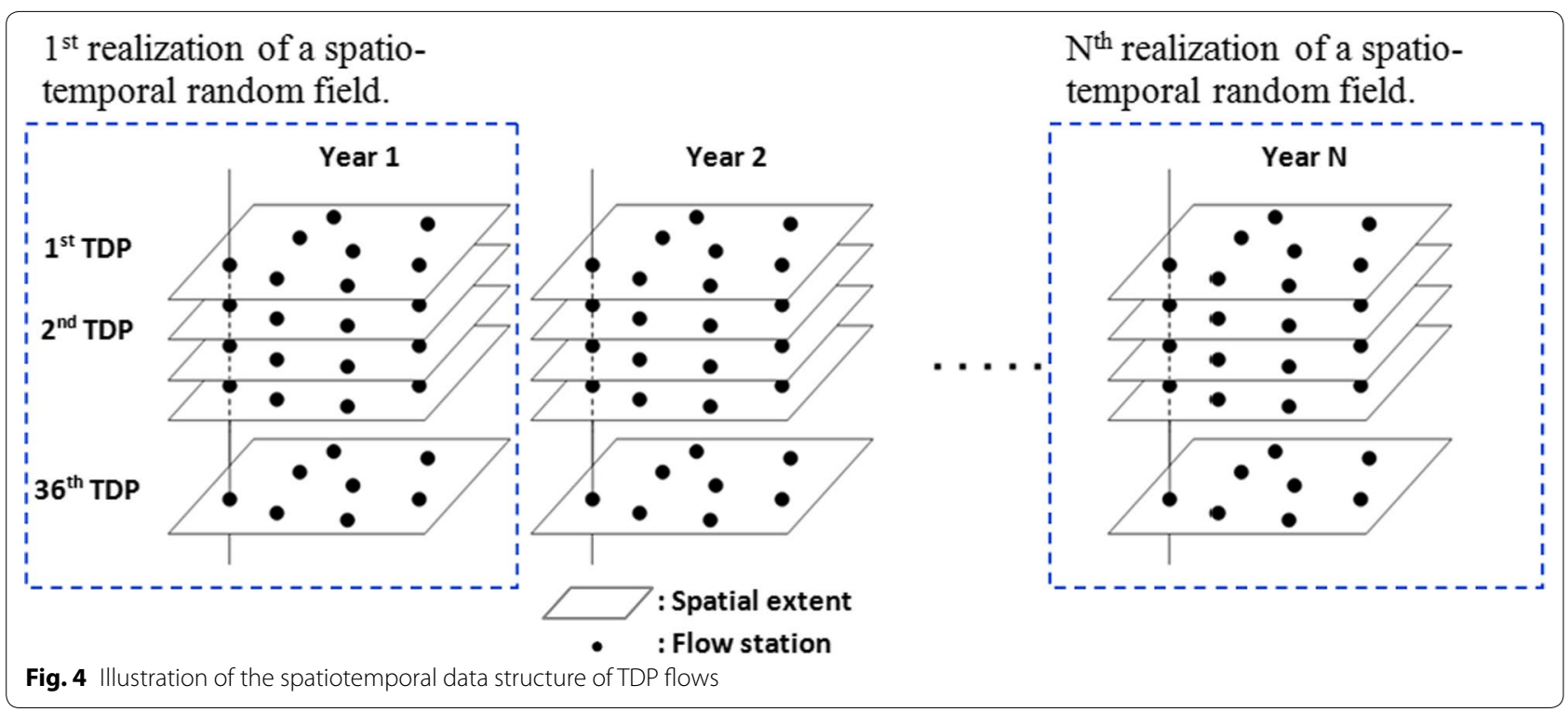

multi-site streamflows which will be described in the next section yielded a large set of such realizations.

The spatiotemporal variation of standardized flows is characterized by two semi-variograms: (1) the spatial semi-variogram $\gamma_{S}(h)$ in the spatial domain and (2) the temporal semi-variogram $\gamma_{T}(t)$ in the temporal domain (in units of TDPs). For a specific TDP of any given year, there are $66(C(12,2))$ spatial pairs of $\left(Z(x), Z\left(x^{\prime}\right)\right)$ within the spatial domain. Similarly, at each station there are $630(C(36,2))$ temporal pairs of $\left(Z(x), Z\left(x^{\prime}\right)\right)$ within the 36-TDP temporal domain. It should be noted that, when considering a spatiotemporal random field, the variable $x$ can represent the locations of flow stations or the ten-day periods depending on the types (spatial or temporal) of semi-variogram modeling.

The spatial semi-variogram $\gamma_{S}(h)$ was constructed using a total of $61,776(C(12,2) \times 36 \times 26)$ same-TDP spatial pairs of standardized flows. Similarly, the temporal semi-variogram $\gamma_{T}(t)$ was constructed using a total of $196,560(C(36,2) \times 12 \times 26)$ same-station temporal pairs of standardized flows. The following exponential semivariogram models were fitted to the empirical spatial and temporal semi-variograms:

$$
\begin{aligned}
& \gamma_{S}(h)=1-e^{-h / 54.6} \\
& \gamma_{T}(t)=1-e^{-t / 2.38}
\end{aligned}
$$

Both the spatial and temporal semi-variograms were fitted with an asymptotic value (the sill) of one since the standardized flows have a unit standard deviation. However, the ranges are numerically quite different. The spatial semi-variogram has a range of approximately $165 \mathrm{~km}$ which implies that standardized flows of the same TDP at two flow stations are independent only if the two flow stations are more than $165 \mathrm{~km}$ apart from each other. Similarly, same-station standardized flows of different TDPs are independent only if the time lag is longer than seven TDPs.

\section{Spatiotemporal model building and stochastic simulation of TDP flows}

We have demonstrated that the standardized flows at different flow stations can be represented by a Pearson type III random field with spatial and temporal semivariograms. However, the standardized flows of different TDPs at different stations (for example, standardized flows of the first TDP at Station 1 and standardized flows of the second TDP at Station 3 may also be correlated. Such correlation involves variations in both the spatial and temporal domains and cannot be characterized by either the spatial or temporal semi-variogram alone. Therefore, it is necessary to develop a spatiotemporal semi-variogram in order to fully characterize the spatiotemporal variation of the standardized flow random field. This is achieved by considering an anisotropic semivariogram model.

In geostatistics, a random field is said to be anisotropic if the spatial variation structures or the semi-variograms vary in different directions. Cheng et al. (2000a, b) developed an anisotropic spatial modeling approach for remote sensing image rectification. Since the ranges of the spatial and temporal semi-variograms of standardized flows are numerically different, the spatiotemporal random field of standardized flows is anisotropic. By 
considering the anisotropic variation in the spatial and temporal domains, a unique spatiotemporal semi-variogram can be expressed by

$$
\gamma(h, t)=1-e^{-\frac{\sqrt{k^{2} h^{2}+t^{2}}}{2.38}}
$$

where $k=0.0436$, the anisotropic ratio, is the ratio of ranges of the temporal and spatial semi-variograms. By rescaling variations in the spatial domain using the anisotropic ratio, the multi-site standardized flows can be treated as an isotropic Pearson type III random field with a unique spatiotemporal semi-variogram $\gamma(h, t)$. It is also noted that the spatiotemporal covariance function $C(h, t)$ can also be expressed as

$$
C(h, t)=e^{-\frac{\sqrt{k^{2} h^{2}+t^{2}}}{2.38}}
$$

Although random field simulation has been widely applied to many studies (Franco et al. 2006; Guillot 1999; Herrick et al. 2002), many of such applications used Gaussian random field generators to generate large sets of realizations which can be characterized by the desired Gaussian distribution and spatial variation structure (or semi-variogram). However, many hydrological and environmental variables are asymmetric and cannot be modeled as Gaussian random fields. Liou et al. (2011) proposed a covariance matrix transformation method for an isotropic non-Gaussian random field simulation in a $2 \mathrm{D}$ spatial domain. This study deals with variations of streamflows in both the temporal and spatial domains. Thus, an anisotropic spatiotemporal semi-variogram model was introduced into the method of Liou et al. (2011) to achieve the anisotropic stochastic simulation of multi-site streamflows. Figure 5 illustrates the conceptual process for stochastic simulation of a Pearson type III random field. The whole process is composed of three sequential components:

1. converting the covariance function $\left(C_{Z}(h)\right)$ of a Pearson type III random field $Z(x)$ to the covariance function $\left(C_{W}(h)\right)$ of a corresponding standard Gaussian random field $W(x)$,

2. generating realizations of the standard Gaussian random field with covariance function $C_{W}(h)$ using a sequential Gaussian simulation (SGS), and

3. transforming realizations of $W(x)$ to corresponding realizations of the Pearson type III random field $Z(x)$.

In a sequential random field simulation process, the covariance function appears as a covariance matrix $\Sigma$ which involves a target point for random number generation and its neighboring points having simulated data. Thus, conversion between the covariance functions
Given a Pearson type III random field $Z(x)$ with known density function parameters and covariance function $C_{Z}(h)$ or semivariogram $\gamma_{Z}(h)$.

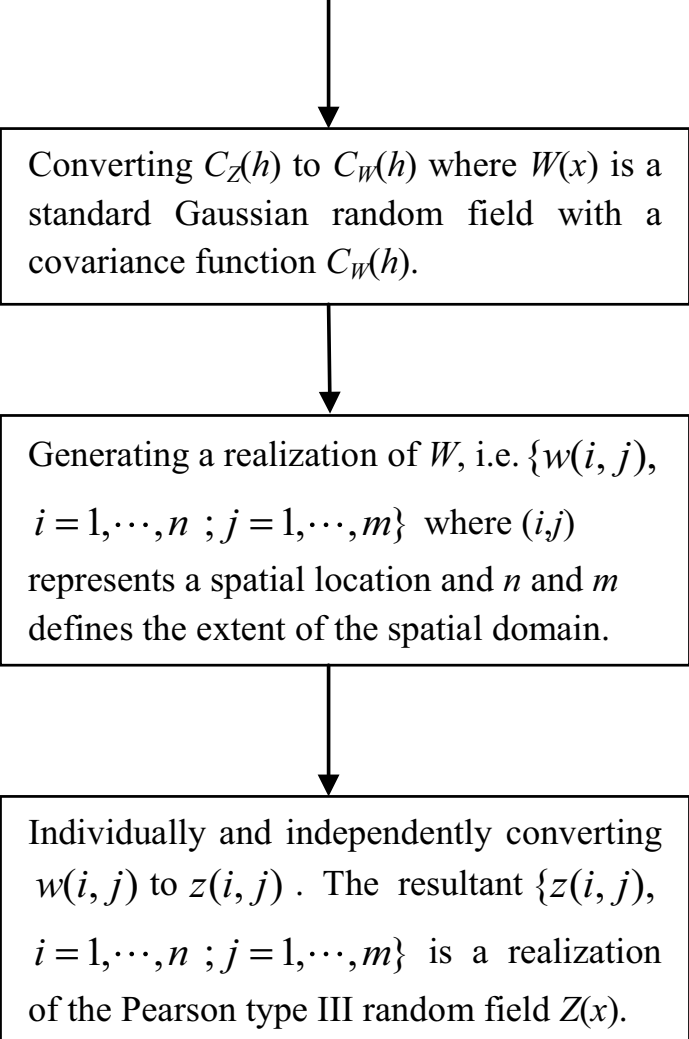

Fig. 5 Conceptual flowchart for stochastic simulation of a Pearson type III random field

$C_{Z}(h)$ and $C_{W}(h)$ is equivalent to conversion between the covariance matrices $\Sigma_{Z}$ and $\Sigma_{W}$ (Liou et al. 2011). Since the multi-site standardized flows are modeled as a Pearson type III random field, conversion between covariance matrices of the Pearson type III random field and the standard Gaussian random field (i.e., $\Sigma_{Z}$ and $\Sigma_{W}$, respectively) is needed for subsequent sequential Gaussian simulation. It is emphasized that an element in $\Sigma_{Z}$ represents the covariance of two random variables of standardized flows in the spatiotemporal domain. For example, let $Z_{1}$ and $Z_{2}$ be, respectively, the standardized flows of the $i$-th TDP at station $s_{1}$ and the standardized flows of the $j$-th TDP at station $s_{2}$. Covariance between $Z_{1}$ and $Z_{2}$ is then calculated as

$\operatorname{Cov}\left(Z_{1}, Z_{2}\right)=C_{Z}\left(h=\left|s_{1}-s_{2}\right|, \quad t=|i-j|\right)=e^{-\frac{\sqrt{k^{2} h^{2}+t^{2}}}{2.38}}$ 
Using Eq. (29), the covariance matrix $\Sigma_{Z}$ can be constructed. It is important to note that each element in $\Sigma_{Z}$ corresponds to an element in $\Sigma_{W}$ which represents the covariance (or correlation) of a bivariate $\left(W_{1}\right.$ and $\left.W_{2}\right)$ standard Gaussian distribution. Conversion between the two bivariate covariance matrices $\left(\Sigma_{Z}\right.$ and $\left.\Sigma_{W}\right)$ can be achieved using the following equation (Cheng et al. 2011):

$\rho_{Z_{1} Z_{2}} \approx\left(A^{2}-6 A C+9 C^{2}\right) \rho_{W_{1} W_{2}}+2 B^{2} \rho_{W_{1} W_{2}}^{2}+6 C^{2} \rho_{W_{1} W_{2}}^{3}$

where $\rho_{Z_{1} Z_{2}}$ and $\rho_{W_{1} W_{2}}$ are, respectively, the correlation coefficients of the bivariate Pearson type III distribution and the bivariate standard Gaussian distribution and $\gamma$ represents the coefficient of skewness of the Pearson type III distribution. The constants $A, B$, and $C$ are defined by

$$
A=1+\left(\frac{\gamma}{6}\right)^{4}, B=\frac{\gamma}{6}-\left(\frac{\gamma}{6}\right)^{3}, C=\frac{1}{3}\left(\frac{\gamma}{6}\right)^{2}
$$

Once the covariance matrix of the standard Gaussian random field is obtained, sequential Gaussian simulation is conducted to generate realizations of the standard Gaussian random field (Liou et al. 2011). Our sequential Gaussian simulation is depicted in Fig. 6. The spatial domain consists of 12 flow stations and the temporal domain comprises 36 TDPs. Each simulation run yields a set of $432(12 \times 36)$ standard Gaussian variates. The simulation is conducted following a column-preference manner, and only points in the spatiotemporal neighborhood of the target point defined by the ranges in the spatial and temporal domains need to be considered for random number generation.

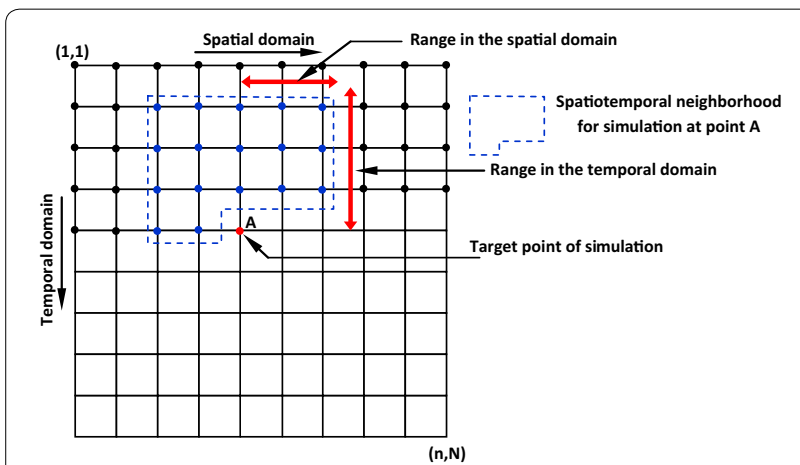

Fig. 6 Illustration of a sequential column-preference generation algorithm for stochastic simulation in a spatiotemporal domain. Only points in the spatiotemporal neighborhood of the target point defined by the ranges in the spatial and temporal domains need to be considered for random number generation at the target point A. Note that the spatiotemporal neighborhood changes with the target point. Parameters $(\Theta)$ include mean, standard deviation, and coefficient of skewness of site- and TDP-specific TDP flows and the spatial and temporal correlation structure (a correlation matrix P of dimension $288 \times 288$ ) of TDP flows
Realizations of the standard Gaussian random field were then transformed to realizations of the multi-site standardized TDP flows through the following equation (Cheng et al. 2011):

$$
\begin{aligned}
z & \approx w+\left(w^{2}-1\right) \frac{\gamma}{6}+\frac{1}{3}\left(w^{3}-6 w\right)\left(\frac{\gamma}{6}\right)^{2} \\
& -\left(w^{2}-1\right)\left(\frac{\gamma}{6}\right)^{3}+w\left(\frac{\gamma}{6}\right)^{4}-\frac{1}{3}\left(\frac{\gamma}{6}\right)^{5}
\end{aligned}
$$

In the above equation, which is the same as the frequency factor equation of the Pearson type III distribution, $w$ represents a simulated standard Gaussian variate in the 36-TDP and 12-site spatiotemporal random field and $z$ is the corresponding standardized flow. Finally, the multi-site TDP streamflows were obtained from $z$ using Eq. (1).

\section{Results and discussion}

In this study, the proposed spatiotemporal simulation model was implemented for TDP streamflow generation at eight flow stations (Stations 1, 3, 4, 7, 8, 9, 10, and 11) with water intake structures. These stations have completely separated tributaries and no station is in the upstream of the other. A single simulation run yields streamflows of 36 TDPs (in 1 year) at each individual station. Since historical flow data are available for a period of 26 years, a total of 26 independent simulation runs are considered as a block-simulation run. A set of parameters including the mean, standard deviation, and coefficient of skewness of the site- and TDP-specific streamflows and their spatial and temporal correlation structure $(\mathrm{P})$ can then be estimated from the results of a block-simulation run (see Fig. 7). These parameter estimates can then be used for performance assessment of the proposed spatiotemporal simulation approach. The spatial and temporal correlation structure is represented by a spatiotemporal correlation matrix $\mathrm{P}$ of dimension $288 \times 288$. The correlation matrix $\mathrm{P}$ is defined as

$$
P=\left[\begin{array}{cccc}
P_{36,1} & P_{36,2} & \cdots & P_{36,36} \\
\vdots & \vdots & & \vdots \\
P_{2,1} & P_{2,2} & \ddots & P_{2,36} \\
P_{1,1} & P_{1,2} & \cdots & P_{1,36}
\end{array}\right]
$$

where $P_{i j}$ itself is a sub-correlation matrix of dimension $8 \times 8$ which represents the correlation between the $i$-th TDP flows and the $j$-th TDP flows at any two sites, i.e.,

$$
P_{i j}=\left[\begin{array}{cccc}
\rho_{8,1}(i, j) & \rho_{8,2}(i, j) & \cdots & \rho_{8,8}(i, j) \\
\vdots & \vdots & & \vdots \\
\rho_{2,1}(i, j) & \rho_{2,2}(i, j) & \ddots & \rho_{2,8}(i, j) \\
\rho_{1,1}(i, j) & \rho_{1,2}(i, j) & \cdots & \rho_{1,8}(i, j)
\end{array}\right]
$$




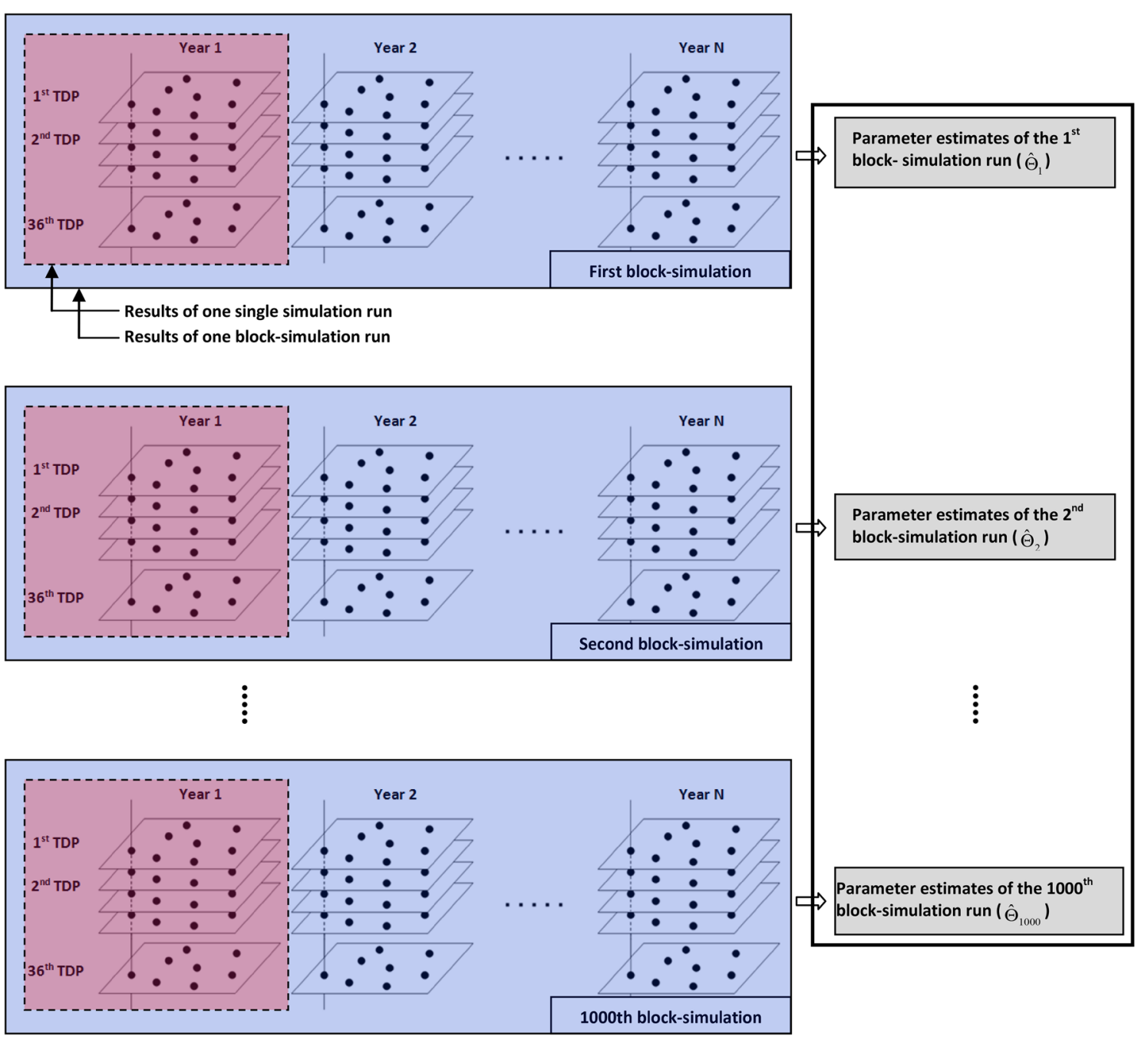

Fig. 7 Illustration of a single simulation run and a block-simulation run. A set of parameters can be estimated from the results of each block-simulation run. Estimates of parameters of the 1000 block-simulation runs can be used for assessment of uncertainties in parameter estimation

In the above equation, $\rho_{m n}(i, j)$ represents the correlation coefficient between streamflows of the $i$-th TDP at the $m$-th station and streamflows of the $j$-th TDP at the $n$-th station.

In order to assess the uncertainties in parameter estimation, a total of 1000 block-simulation runs were conducted in our study. Spatiotemporal variation and statistical properties of estimates of the parameters (including the mean, standard deviation, and coefficient of skewness) of standardized flows are shown in Fig. 8. In each panel of Fig. 8, the subpanel at the top represents parameter estimates based on one arbitrarily chosen block-simulation run, and subpanels in the middle and at the bottom represent the mean and standard deviation of the parameter estimates based on 1000 block-simulation runs, respectively. Parameter estimates based on a particular block-simulation run are considered as sample estimates and can exhibit higher degree of spatial and temporal variations. In contrast, the means of parameter estimates based on 1000 block-simulation runs are close to their theoretical (or model) values (0, 1, and 2.3167 for the mean, standard deviation, and coefficient of skewness, respectively) with minor variation in the spatial and temporal domains. Figure 8 also demonstrates that the uncertainties of parameter estimates, which are measured by their standard deviations, are nearly uniform across the temporal and spatial domains. The above assessments indicate that realizations generated by the proposed spatiotemporal simulation approach can preserve the statistical properties of the marginal density.

As for the assessment of the spatiotemporal correlation matrix, Fig. 9 shows the $\mathrm{P}$ matrix derived from 

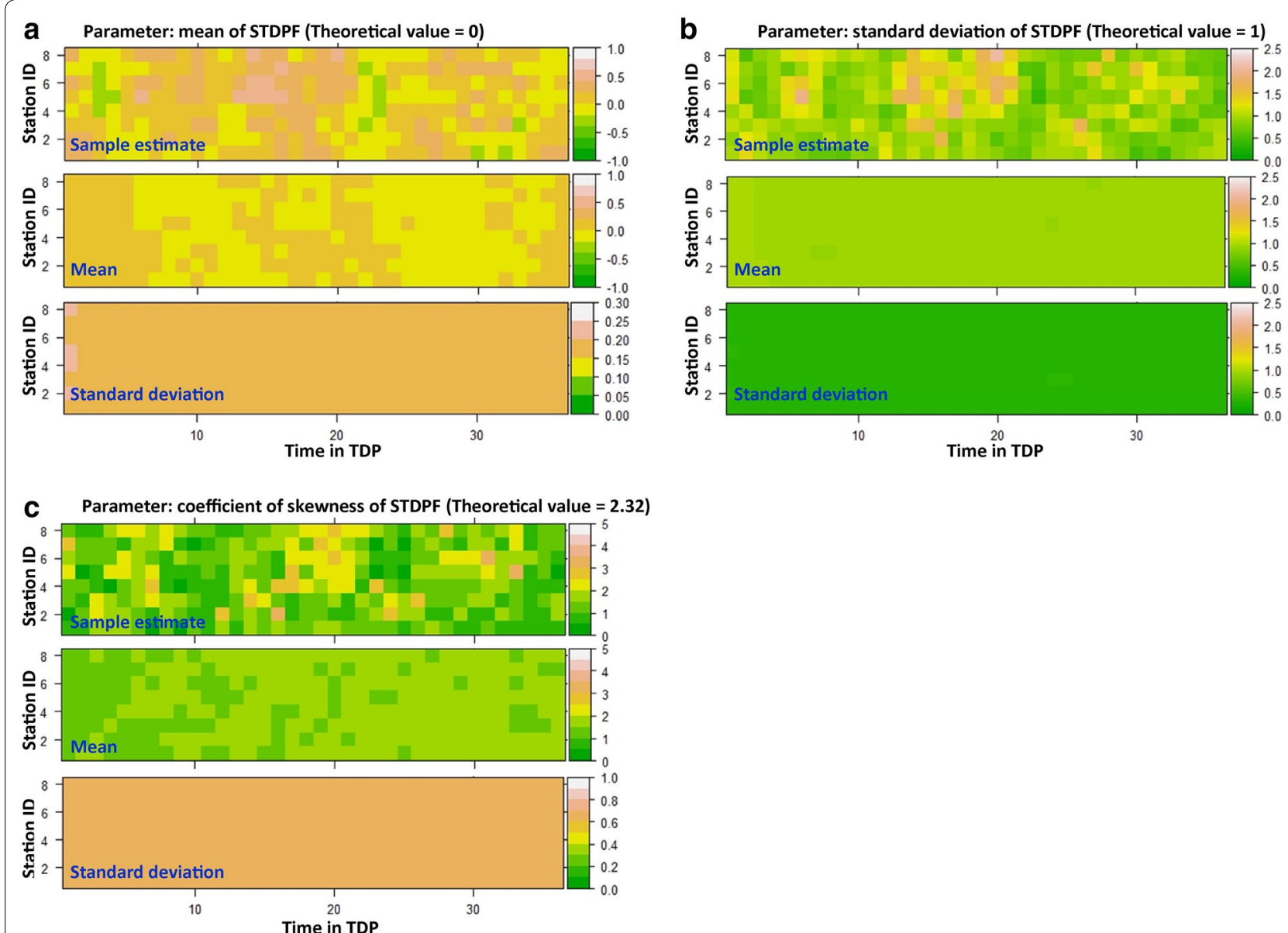

Fig. 8 Spatial and temporal variations of estimates of parameters (mean, standard deviation, and coefficient of skewness) of standardized ten-day period flows (STDPF)

26 years of historical multi-site TDP flows [panel (a)] and empirical spatiotemporal correlation matrices based on six different block-simulation runs [panels (d)-(i)]. The mean and standard deviation of the empirical $\mathrm{P}$ matrices of the 1000 block-simulation runs are also shown in panels (b) and (c) of Fig. 9, respectively. It demonstrates that the proposed spatiotemporal stochastic simulation model is capable of generating multi-site TDP flows with spatiotemporal correlation pattern similar to that of the observed multi-site TDP flows.

From the results of 1000 block-simulation runs, the $95 \%$ acceptance interval of any element $\rho_{m n}(i, j)$ in the spatiotemporal correlation matrix (P) can be estimated using the 2.5 and $97.5 \%$ quantiles of $\rho_{m n}(i, j)$ estimates. Three profiles (namely profiles 40, 144, and 200) of the spatiotemporal correlation matrix [see panel (a) of Figs. 9, 10] were selected to quantitatively assess the spatiotemporal correlation structure of the proposed model. Figure 10 depicts that the empirical spatiotemporal correlation coefficients derived from the historical TDP flows mostly fall within the $95 \%$ acceptance intervals, with only a few exceptions in April and late September. Such results indicate that the proposed model can capture the spatiotemporal variation characteristics of the multi-site TDP flows in our study area. Results of TDP flow simulation at eight stations with water intake structures can be used for assessing the effect of certain water management measure on reducing the risk of irrigation water shortage. An exemplary application is demonstrated below.

The JID is divided into four irrigation management districts (IMDs). IMD-1 consists of streamflows from Bei-Gun River, Pu-Tz River, and Bah-Jon River. IMD-2 withdraws water from the Tzi-Shuei River. IMD-3 receives irrigation water from the Tseng-Wen Reservoir. IMD-4 receives water from the Yen-Shuei River. Currently, water conveyance between the IMD-1 and IMD-2 is not possible since there are no irrigation channels connecting the two districts. The IMD- 1 has significantly higher flows than IMD-2 and thus it is desired to convey additional flows from IMD-1 to IMD-2 in case of drought 

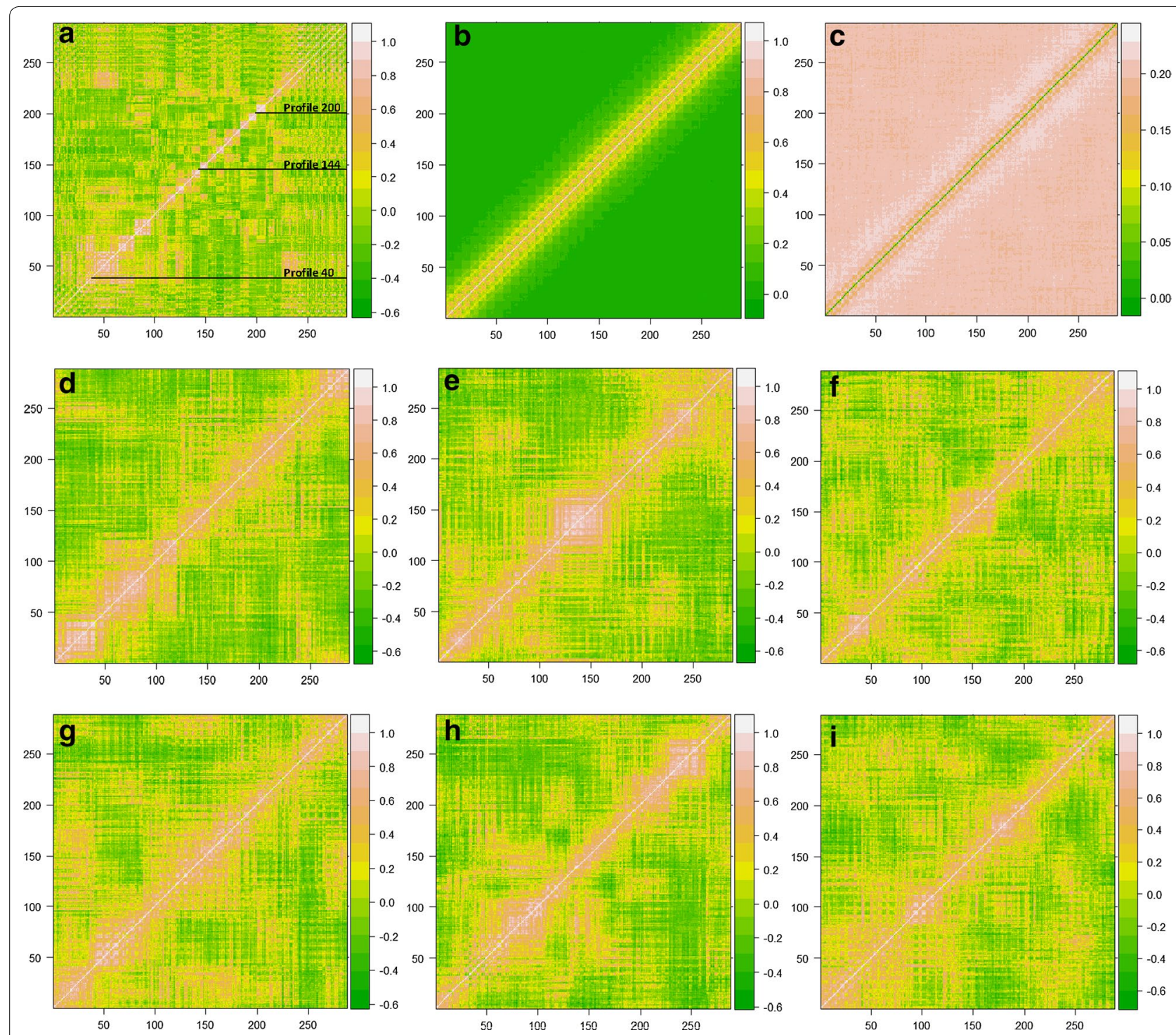

Fig. 9 Spatiotemporal correlation structure of flows. a Spatiotemporal correlation matrix P derived from 26 years of historical multi-site flows. b, c Mean and standard deviation of P matrices of 1000 block-simulation runs, respectively. $\mathbf{d}$-i Spatiotemporal correlation matrices of different blocksimulation runs. Details of the three correlation profiles in panel (a) are shown in Fig. 10

occurrences. In this exemplary application, we investigate changes in the risk of irrigation water shortage, if the mitigation measure of establishing water conveyance between the two districts is implemented.

One thousand runs of spatiotemporal streamflow simulation were conducted for this example application study. Each simulation run yields a spatiotemporal sample consisting of 36 TDP streamflows at each flow station. Using the results of each simulation run, an irrigation management model calculated the amounts of irrigation water that can be provided to individual IMDs under two different situations-(1) existing situation (without water conveyance between IMD-1 and IMD-2) and (2) with the mitigation measurement implemented. A detailed description of the irrigation management is out of the scope of this study and readers are referred to Wen et al. (2007) for details of the irrigation management. Irrigation water demands vary from one TDP to another within crop growth periods and the ratio of irrigation water shortage (hereinafter referred to as the shortage ratio) is defined as:

$$
r(t)=\left\{\begin{array}{lll}
{\left[X_{d}(t)-X_{s}(t)\right] / X_{d}(t),} & \text { if } & X_{d}(t)>X_{s}(t) \\
0, & \text { if } & X_{d}(t) \leq X_{s}(t)
\end{array}\right.
$$

where $X_{d}(t)$ and $X_{s}(t)$, respectively, represent the amounts of irrigation demand and irrigation water supply of the $t$ th TDP. Not only does the shortage ratio vary with TDPs 

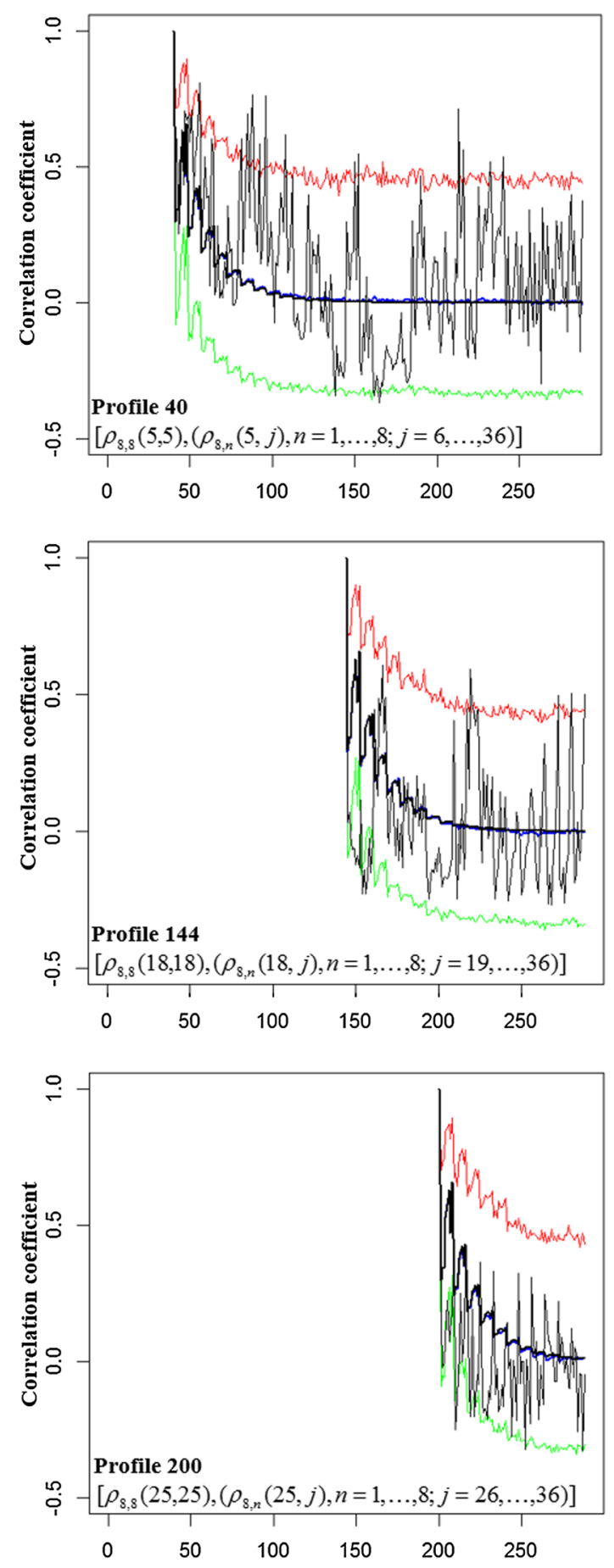

Theoretical (model) correlation profile

Mean profile based on 1000 block-simulation runs.

Upper bound of the $95 \%$ interval

Lower bound of the $95 \%$ interval

Empirical correlation profile based on historical data.

Column index of the spatiotemporal correlation matrix

Fig. 10 Comparison of the theoretical correlation profile and the empirical correlation profile derived from multi-site historical TDP flows. See panel (a) in Fig. 9 for locations of three profiles in the empirical correlation matrix

in 1 year but also from 1 year (simulation run) to another. Thus, from the results of 1000 spatiotemporal simulation runs, average TDP-specific shortage ratios with and without implementation of the water conveyance measure can be estimated. Figure 11 illustrates that, with implementation of the mitigation measure, the shortage ratios of IMD-2 can be significantly reduced during the 4-12 TDP period (first crop) and the 26-30 TDP period (second 


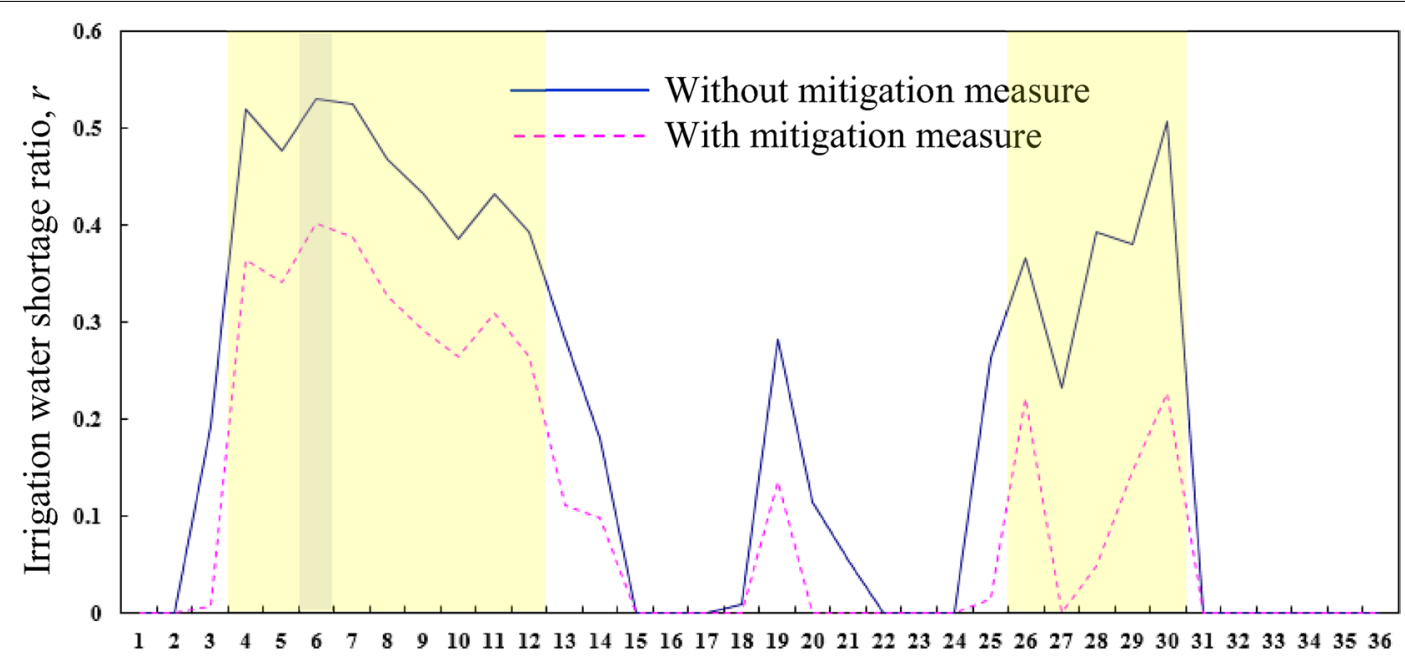

Time, TDP

Fig. 11 Irrigation water shortage ratios of IMD-2, with and without implementation of the mitigation measure. Risks of irrigation water shortage for the 6th TDP are illustrated in Fig. 12

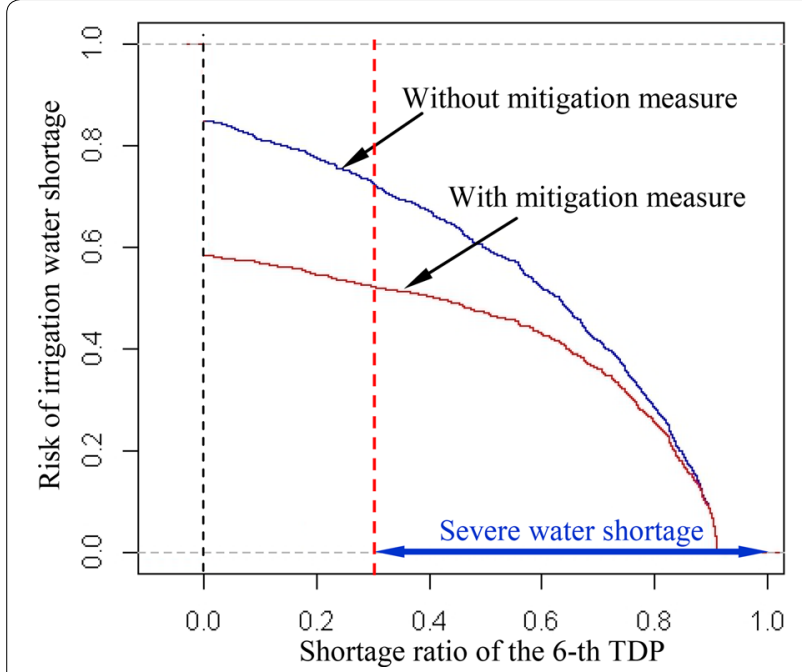

Fig. 12 Risks of irrigation water shortage for the 6th TDP with and without implementation of the mitigation measure

crop). On average, reductions in the shortage ratios are 0.13 and 0.25 for the first and second crop periods, respectively.

Additionally, for any specific TDP, the probability of the shortage ratio being higher than 0 is defined as the risk of irrigation water shortage in this study. With this definition, we could then assess the risks of irrigation water shortage for IMD-2 under the current situation and with implementation of the mitigation measure. Such assessments can be conducted with regard to individual TDPs. Taking the 6th TDP as an example, the probability of experiencing irrigation water shortage (shortage ratio $>0$ ) in IMD- 2 is 0.83 and 0.58 for the current situation and the situation with proposed mitigation measure, respectively. The mitigation measure is able to reduce the risk of irrigation water shortage in IMD-2 for the 6th TDP by 0.25 . We can also consider the risk of more severe irrigation water shortage. For example, shortage ratio exceeding 0.3 is considered as severe water shortage in the JID and may prompt reduced water supply. By implementing the proposed mitigation measure, the risk of severe water shortage in IMD-2 for the 6th TDP can be reduced from 0.72 to 0.52 . Thus, the proposed spatiotemporal simulation approach not only can take into account the spatial and temporal variations/co-variations of the multi-site streamflows but also facilitates probabilistic assessment of effectiveness of drought mitigation measures. As a final note, we like to emphasize that it is possible to comparatively evaluate the effectiveness of different mitigation measures by considering the overall reduction in the risk of the severe water shortage during the crop growth period.

\section{Conclusions}

A stochastic simulation model capable of characterizing the spatiotemporal variability of multi-site streamflows was developed and validated using historical flow data in the Jianan Irrigation District. We also demonstrate that the spatiotemporal flow simulation results can be used for assessing the effect of certain water management measure on reducing the risk of irrigation water shortage. A few concluding remarks are made as follows: 
1. Spatiotemporal random field can be used to characterize streamflows at different locations and their temporal variations. A spatiotemporal anisotropic semi-variogram is required to fully characterize the spatiotemporal variation of the standardized flow random field.

2. Spatiotemporal flow simulation can generate a large number of realizations which preserve not only the marginal distributions of streamflows at individual stations, but also the spatial and temporal correlations of streamflows. Such simulated realizations can be used to assess the effect of certain water management measure on reducing the risk of irrigation water shortage, as demonstrated in this paper.

3. For the Jianan Irrigation District, by implementing the mitigation measure of conveying additional water from IMD-1 to IMD-2, the irrigation water shortage ratios can be reduced by 0.13 and 0.25 for the first and second crop periods, respectively. The risk of severe water shortage can also be effectively reduced.

\section{Authors' contributions}

KSC conceived the stochastic modeling and simulation part of the study, devised the simulation algorithm, participated in data and result interpretation, and drafted the manuscript. MDS conceived the irrigation risk assessment and mitigation measure part of the study and participated in data and result interpretation. $\mathrm{HIH}$ collected the data, wrote computer code, and carried out the stochastic simulation. YCW wrote computer code for streamflow goodness-of-fit tests and carried out the L-moments-based goodness-of-fit tests. All authors discussed the results and implications and commented on the manuscript at all stages. All authors read and approved the final manuscript.

\section{Authors' information}

Hsin-I Hsieh is currently a Postdoctoral Research Associate at the Department of Bioenvironmental Systems Engineering, National Taiwan University. She received B.Sc. (2005), M.Sc. (2007), and Ph.D. (2014) degrees from National Taiwan University, Taipei, Taiwan. Her studies focus on irrigation and water resources management, spatial analysis, and linear programming and its applications.

Ming-Daw Su is currently a Professor with the Department of Bioenvironmental Systems Engineering, National Taiwan University, and also acts as a Director of Agricultural Engineering Research Center at Chung-Li, Taiwan. He received his B.Sc. degree (1976) from National Taiwan University, Taipei, Taiwan, and M.Sc. (1979) and Ph.D. (1989) degrees from the Utah State University, Logan, UT, USA. His research interests focus on irrigation and water resources management, natural hazard risk analysis, and spatial data analysis and its applications.

Yii-Chen Wu is a Postdoctoral Research Associate at the Department of Bioenvironmental Systems Engineering. She received her B.Sc. degree (2003) from the National Pingtung University of Science and Technology, Pingtung, Taiwan, and M.Sc. (2005) and Ph.D. (2011) degrees from the National Taiwan University, Taipei, Taiwan. She specializes in hydrological modeling and design, stochastic simulation, and hydrological frequency analysis.

Ke-Sheng Cheng is currently a Professor with the Department of Bioenvironmental Systems Engineering, National Taiwan University. He received his B.Sc. and M.Sc. degrees from National Taiwan University, Taipei, Taiwan, in 1979 and 1983, respectively, and Ph.D. degree from the University of Florida, Gainesville, FL, USA, in 1989. His research interests focus on three interrelated fields: hydrologic modeling and forecasting, environmental remote sensing, and spatial and geostatistical modeling. He is particularly interested in applying stochastic modeling and simulation to environmental processes and climate change.

\section{Author details}

${ }^{1}$ Department of Bioenvironmental Systems Engineering, National Taiwan University, Taipei, Taiwan. ${ }^{2}$ Master Program in Statistics, National Taiwan University, Taipei, Taiwan.

\section{Acknowledgements}

We acknowledge the financial support provided by the Council of Agriculture (COA) of Taiwan through Project 101 AgriSci-8.2.5-WRE-b1(3).

\section{Competing interests}

The authors declare that they have no competing interests.

Received: 16 February 2015 Accepted: 22 January 2016

Published online: 09 February 2016

\section{References}

Cheng KS, Yeh HC, Liou CY (2000a) Comparative study of drought prediction techniques for reservoir operation. J Am Water Resources Association 36(3):511-521

Cheng KS, Yeh HC, Tsai CH (2000b) An anisotropic spatial modeling approach for remote sensing image rectification. Remote Sensing Environ 73(1):46-54

Cheng KS, Wei C, Cheng YB, Yeh HC (2003) Effect of spatial variation characteristics on contouring of design storm depth. Hydrological Process 17:1755-1769

Cheng KS, Hou JC, Liou JJ (2011) Stochastic simulation of bivariate gamma distribution-a frequency-factor based approach. Stochastic Environ Res Risk Assessment 25(2):107-122

Fernandez B, Salas JD (1990) Gamma-autoregressive models for stream-flow simulation. J Hydraulic Eng 116(11):1403-1414

Franco C, Soares A, Delgado J (2006) Geostatistical modelling of heavy metal contamination in the topsoil of Guadiamar river margins (S Spain) using a stochastic simulation technique. Geoderma 136:852-864

Greenwood JA, Landwehr JM, Matalas NC, Wallis JR (1979) Probability weighted moments: definition and relation to parameters of several distributions expressible in inverse form. Water Resources Res 15:1049-1054

Guillot G (1999) Approximation of Sahelian rainfall fields with meta-Gaussian random functions. Part 1: model definition and methodology. Stochastic Environ Res Risk Assessment 13:100-112

Herrick MG, Benson DA, Meerschaert MM, McCall KR (2002) Hydraulic conductivity, velocity, and the order of the fractional dispersion derivative in a highly heterogeneous system. Water Resources Res 38(11):1227-1239

Hosking JRM, Wallis JR (1995) A comparison of unbiased and plotting-position estimators of L-moments. Water Resources Res 31 (8):2019-2025

Hosking JRM, Wallis JR (1997) Regional Frequency Analysis: An Approach based on L-moments. Cambridge University Press, Cambridge

Hsieh HI, Su MD, Cheng KS (2014) Multisite spatiotemporal streamflow simulation - with an application to irrigation water shortage risk assessment. Terrestrial, Atmospheric and Oceanic Sci 25(2):255-266. doi:10.3319/ TAO.2013.10.21.01(Hy)

Journel AG, Huijbregts CJ (1978) Mining Geostatistics. Academic Press, London Liou JJ, Wu YC, Cheng KS (2008) Establishing acceptance regions for L-moments-based goodness-of-fit test by stochastic simulation. J Hydrology 355(1-4):49-62

Liou JJ, Su YF, Chiang JL, Cheng KS (2011) Gamma random field simulation by a covariance matrix transformation method. Stochastic Environ Res Risk Assessment 25(2):235-251. doi:10.1007/s00477-010-0434-8

Machiwal D, Jha MK (2006) Time series analysis of hydrologic data for water resources planning and management: a review. J Hydrology Hydromechanics 54(3):237-257

Wen TH, Lin CH, Chen CT, Su MD (2007) Analysis of spatial scenarios aiding decision making for regional irrigation water-demand planning. J Irrigation Drainage Eng 133:455-467. doi:10.1061/ (ASCE)0733-9437(2007)133:5(455)

Wu YC, Liou JJ, Cheng KS (2012) Establishing acceptance regions for L-moments based goodness-of-fit tests for the Pearson type III distribution. Stochastic Environ Res Risk Assessment 26:873-885 